\title{
Overview of LHCb RICH Detector Development
}

\author{
On Behalf of \\ LHCb-RICH Group
}

$\mathrm{RICH} 2004$

Playa Del Carmen, Mexico

December 4, 2004

S. Easo

Rutherford-Appleton Laboratory, U.K.

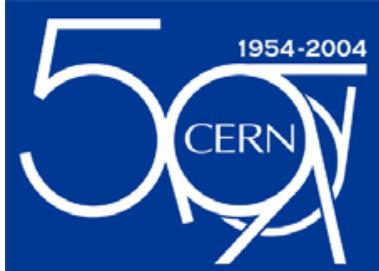




\section{OUTLINE}

- LHCb and its Particle Identification

- Design and Main Features of LHCb-RICH

- Components of RICH1 and RICH2:

Radiators: Aerogel, $\mathrm{C}_{4} \mathrm{~F}_{10}$ gas, $\mathrm{CF}_{4}$ gas.

Mirrors: Beryllium, Glass Type.

- RICH Photodetectors: HPD, Readout System

- RICH Performance : LHCb Detector Simulations

- Summary 


\section{LHCb EXPERIMENT}

- Precision measurements of CP violation in B Meson System

- Search for Signals of 'New Physics' beyond Standard Model

- Large samples of events with $B_{d}$ and $B_{s}$ Mesons

- At the beginning of LHC $2 * 10^{9} \mathrm{~b} \bar{b}$ events per year after trigger selection

- Most of the b-hadrons produced at small polar angles

- Single forward arm spectrometer with open geometry

- From the CP Asymmetries in the final states of $B$ meson decays, measure CKM Angles.

Examples:

$\gamma$ from $\quad \mathrm{B}_{\mathrm{d}}^{0} \rightarrow \pi^{+} \pi^{-}, \mathrm{B}_{\mathrm{s}}^{0} \rightarrow \mathrm{K}^{+} \mathrm{K}^{-}$

$\gamma$ from $\quad \mathrm{B}_{\mathrm{s}}^{0} \rightarrow \mathrm{D}_{\mathrm{s}}^{+} \mathrm{K}^{-}$
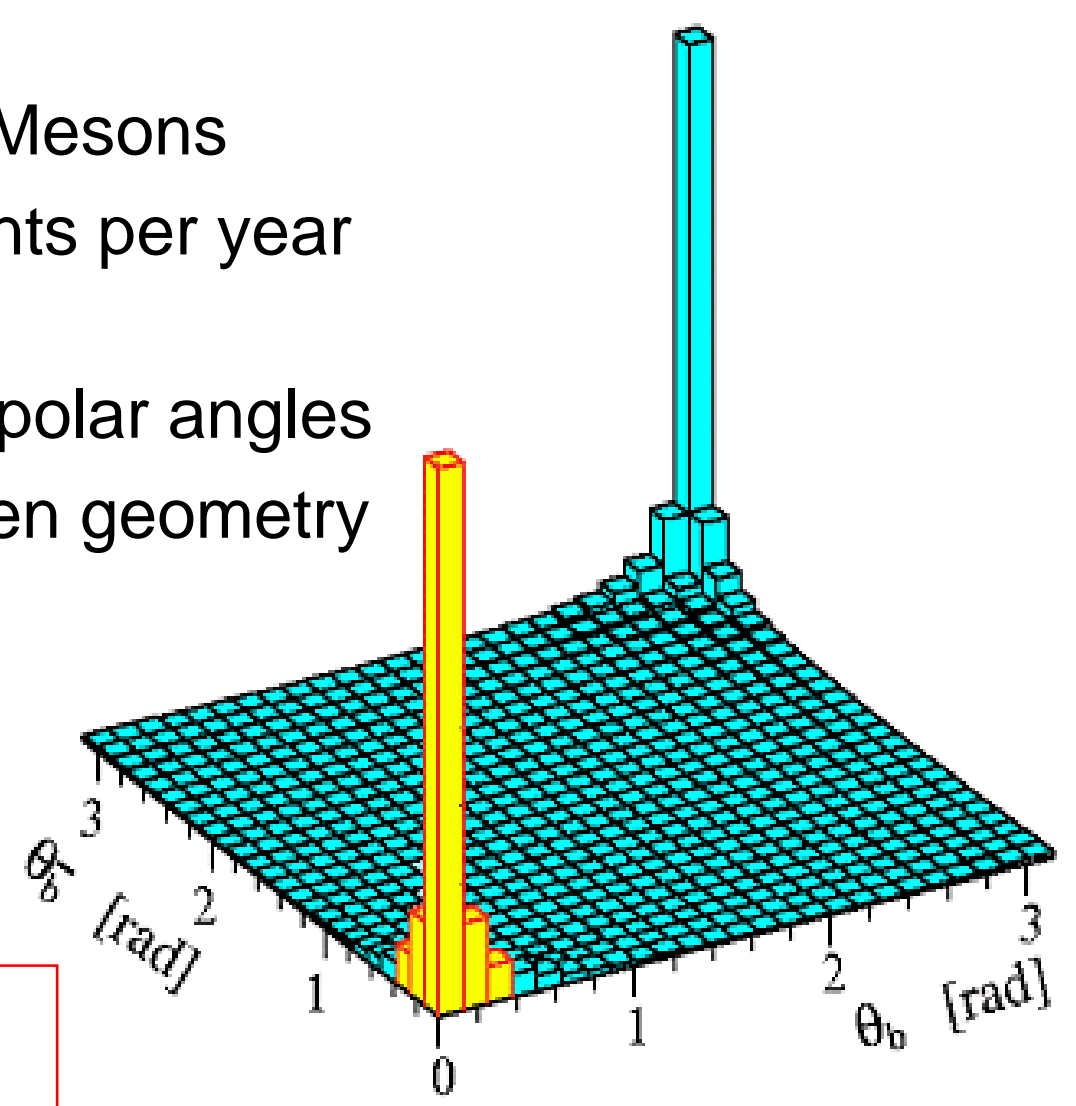


\section{THE LHCb EXPERIMENT}

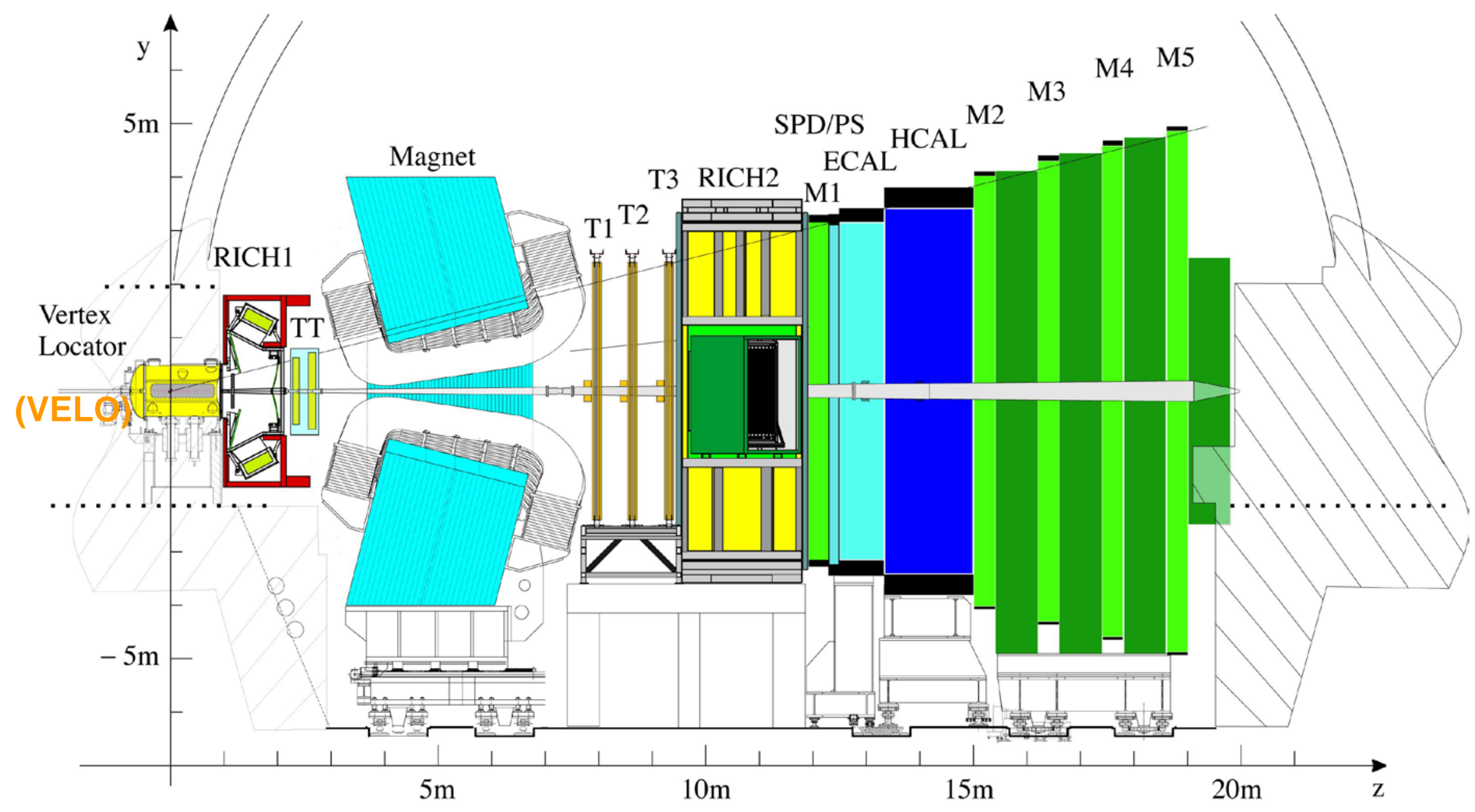

Magnet already installed 


\section{PARTICLE IDENTIFICATION IN LHCb}

Particle Identification using $\mathrm{RICH}$ is an essential part of $\mathrm{LHCb}$.
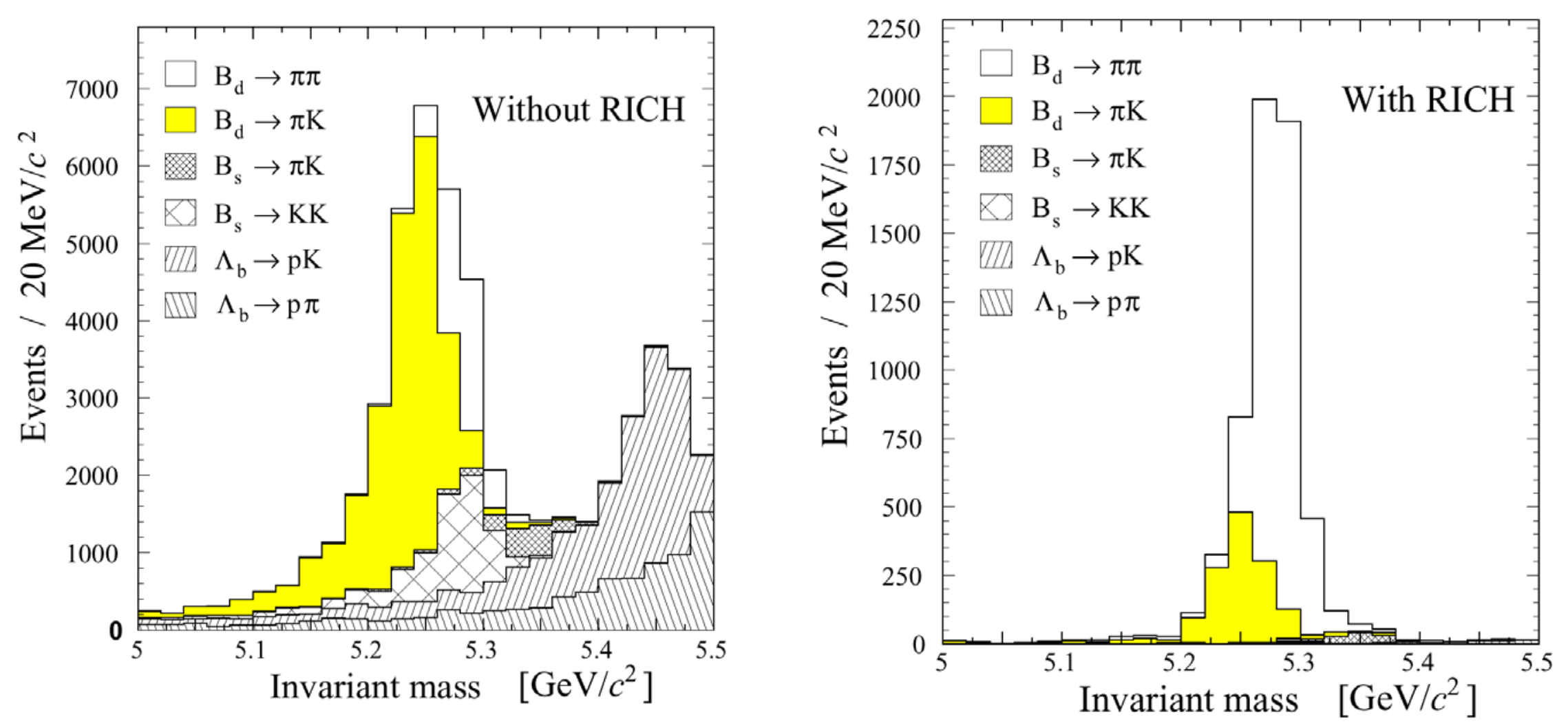

- Identification of Kaons to tag the flavour of $b$ hadrons where $b \rightarrow c \rightarrow s$

- Momentum Range: $2 \rightarrow 100 \mathrm{GeV} / \mathrm{c}:$

- Upper limit from the $\pi$ in $B_{d} \rightarrow \pi^{+} \pi^{-}$

- Lower limit from the tagging Kaons 


\section{LHCb-RICH SPECIFICATIONS}

RICH1: Aerogel L=5cm p: $2 \rightarrow 10 \mathrm{GeV} / \mathrm{c}$ $\mathrm{n}=1.03$ (nominal at $540 \mathrm{~nm}$ )

$\mathrm{C}_{4} \mathrm{~F}_{10} \quad \mathrm{~L}=85 \mathrm{~cm}$ p: $<70 \mathrm{GeV} / \mathrm{c}$ $\mathrm{n}=1.0014$ (nominal at $400 \mathrm{~nm}$ )

Upstream of LHCb Magnet Acceptance: $25 \rightarrow 250$ mrad (vertical) 300 mrad (horizontal)

Gas vessel: $2 \times 3 \times 1 \mathrm{~m}^{3}$

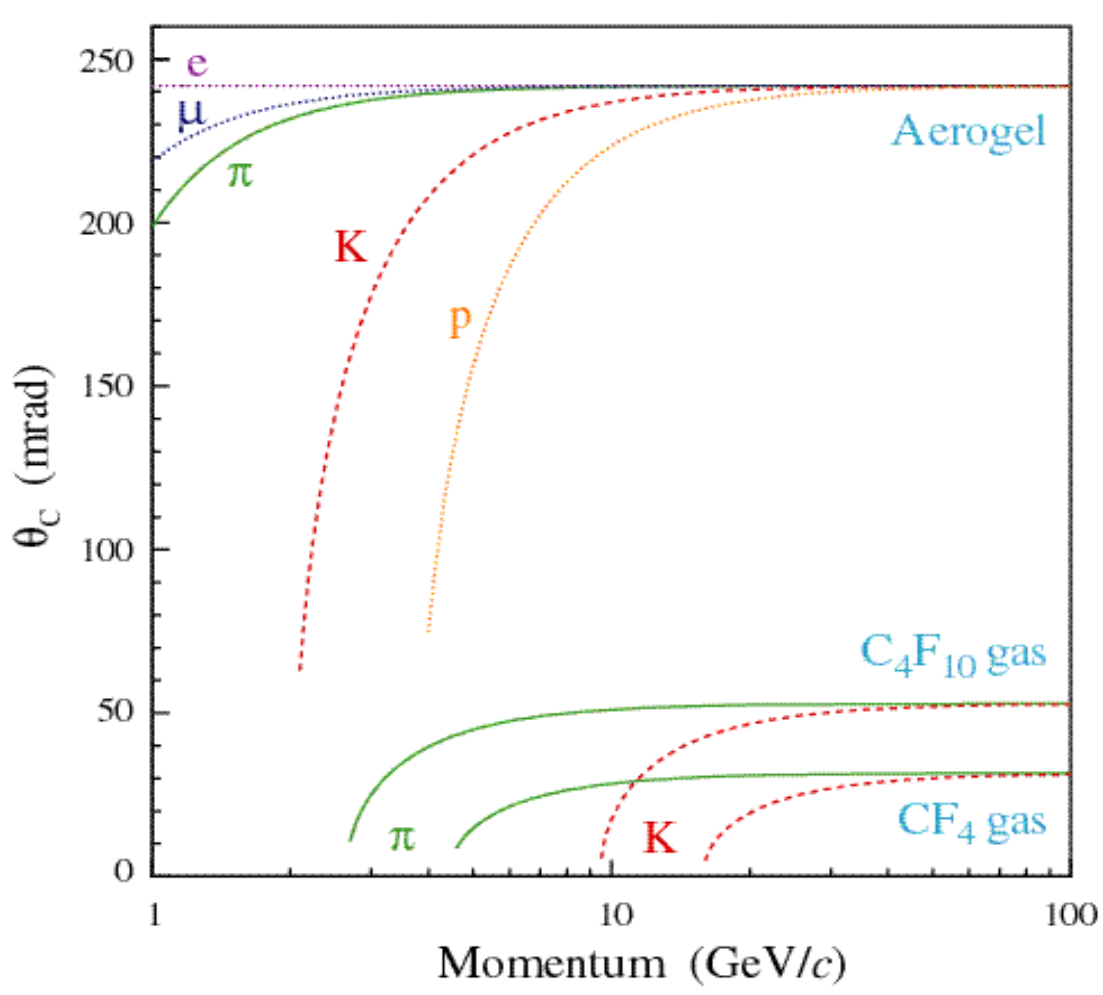

$\theta_{\mathrm{C}} \max$

$242 \mathrm{mrad}$ (a)

$53 \mathrm{mrad}$

$32 \mathrm{mrad}$

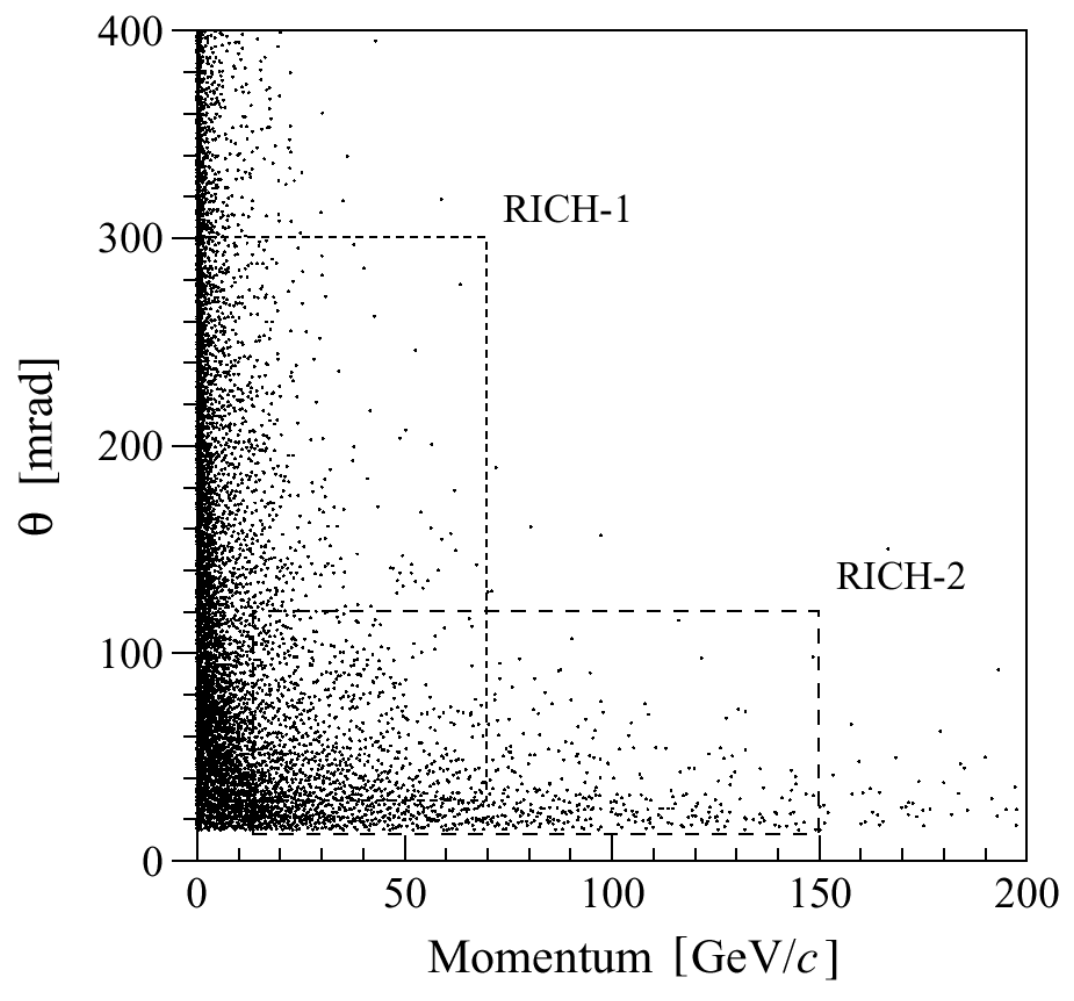

RICH2: CF $_{4} \quad$ L=196 cm p: $<100 \mathrm{GeV} / \mathrm{c}$ $\mathrm{n}=1.0005$ (nominal at $400 \mathrm{~nm}$ )

Downstream of LHCb Magnet Acceptance: $15 \rightarrow 100$ mrad (vertical) $120 \mathrm{mrad}$ (horizontal) 


\section{RICH1 SCHEMATIC}

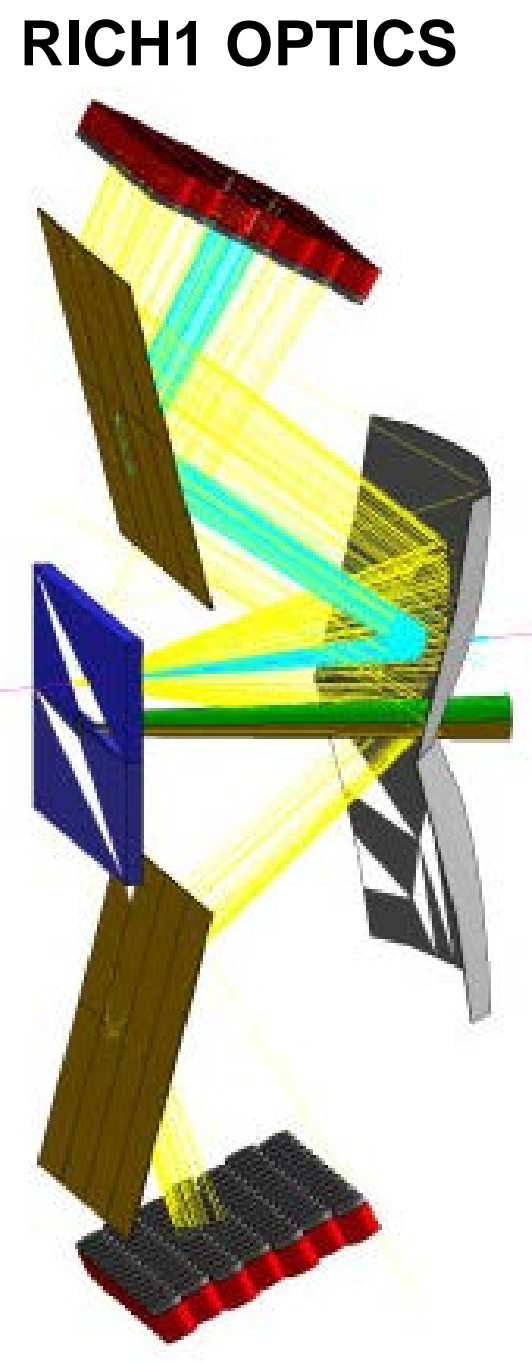

- Spherical Mirror tilted to keep photodetectors outside acceptance (tilt=0.3 rad)

Magnetic Shield

Spherical Mirror

Flat Mirror

Gas Enclosure

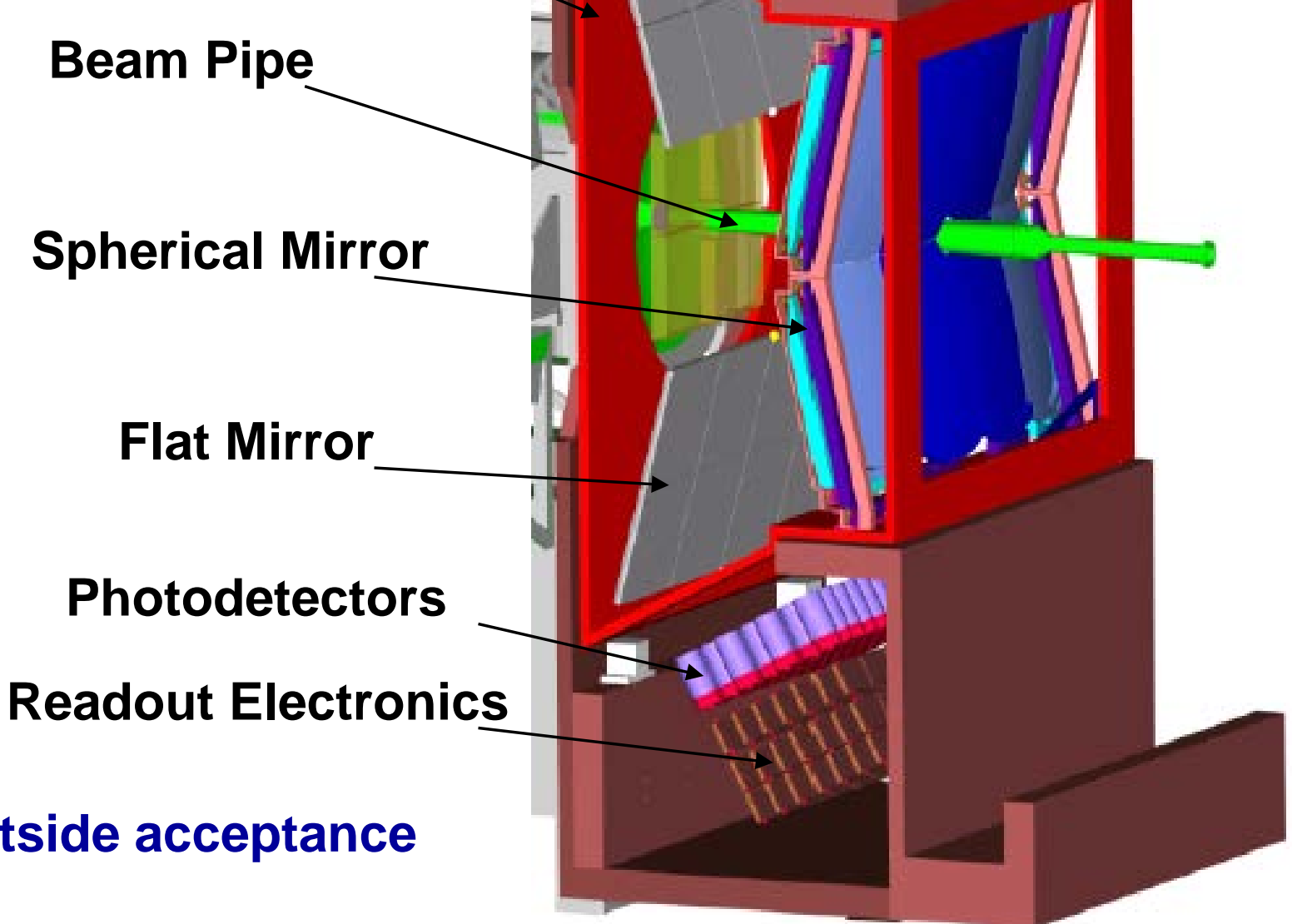




\section{COMPONENTS OF RICH1}

- Gas Enclosure:

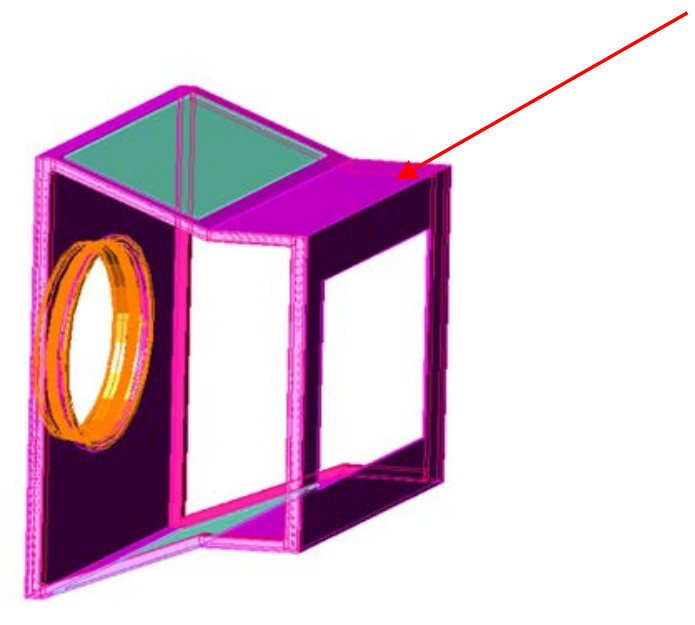

- Aerogel and Mirrors attached to this box

- Non-magnetic, to minimize distortions from the field on the optical configuration

- Made of $30 \mathrm{~mm}$ thick Aluminium alloy

- Exit window:

- Low mass material

- PMI (polymethacrylimide) foam between carbon fibre epoxy

Radiators:

- $\mathrm{C}_{4} \mathrm{~F}_{10}$ : Results with prototypes in testbeam already published

- Concern over availability of $\mathrm{C}_{4} \mathrm{~F}_{10}$ : Backup Option: 50:50 mixture of $C_{5} F_{12}$ and $C_{3} F_{8}$

- Silica Aerogel:

- fragile linked network of $\mathrm{SiO}_{2}$ nanocrystals

- hygroscopic

- nominal n=1.03 at $540 \mathrm{~nm}$

- Loss of signal from Rayleigh Scattering

Ref.Talk by C. Matteuzzi

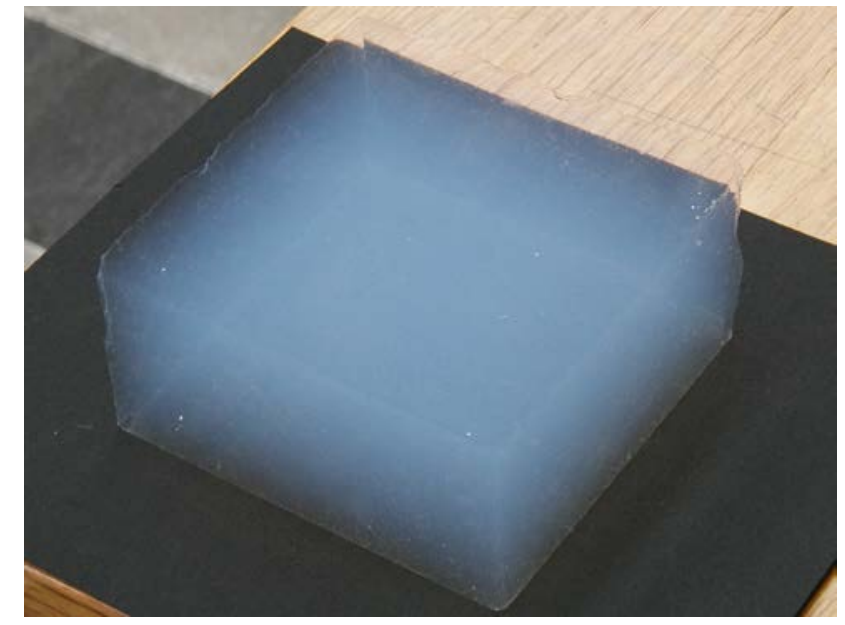




\section{RICH1 MIRRORS}

- Spherical Mirror inside LHCb acceptance

- Requirements:

- Minimum material with sufficient rigidity

- $D_{0}<2.5 \mathrm{~mm}$ (size of circle at focal plane with $95 \%$ image intensity from a point source)

- Reflectivity > 90 \% in 200-700 nm

- Selected $3 \mathrm{~mm}$ thick Beryllium $+<0.3 \mathrm{~mm}$ glass coated with $\mathrm{Al}+\mathrm{SiO}_{2}+\mathrm{HfO}_{2}: 0.8 \% \mathrm{X}_{0}$.

- RoC $=2700 \mathrm{~mm}$

- 8 segments : $410 \times 600 \mathrm{~mm}$ and $385 \times 600 \mathrm{~mm}$

- $D_{0}=0.41 \mathrm{~mm}$ for prototype, FEA: negligible distortions

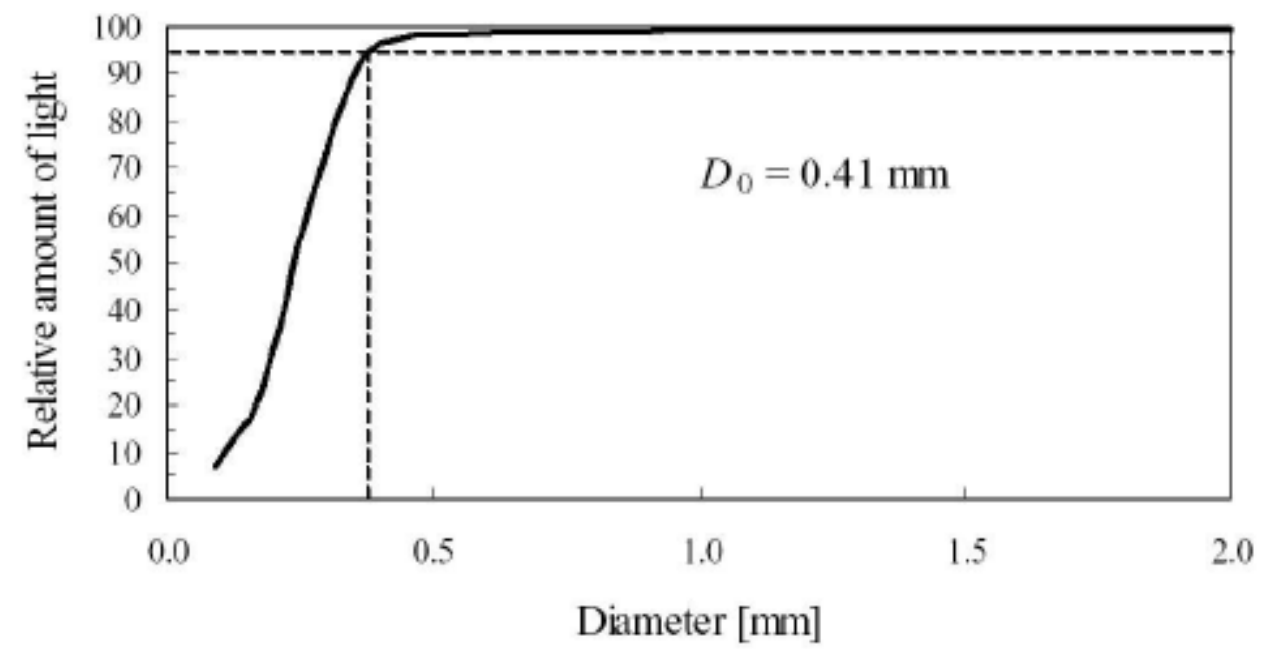

- Flat Mirror outside LHCb acceptance:

- 16 segments : 370 X 387 mm

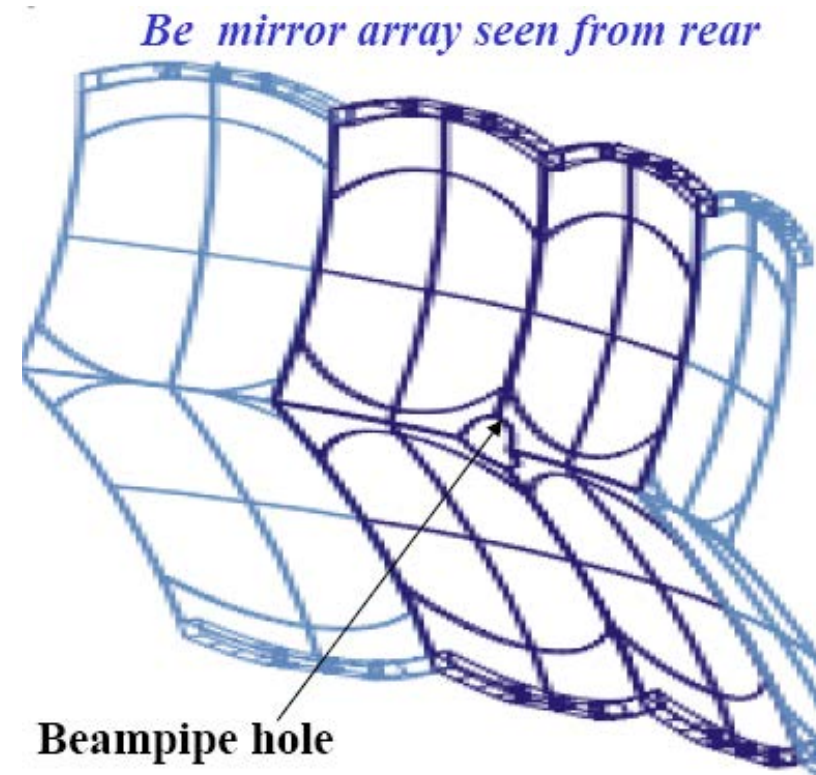

- 6 mm thick Simax (Borosilicate) glass or equivalent

Be Prototype

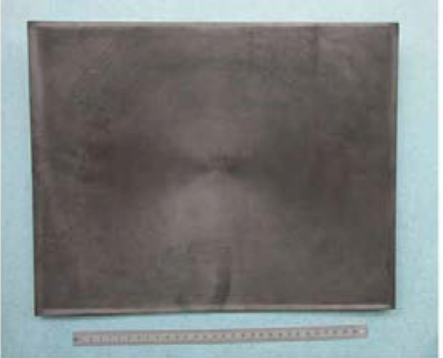




\section{RICH2 SCHEMATIC}

RICH2 Optics Top View

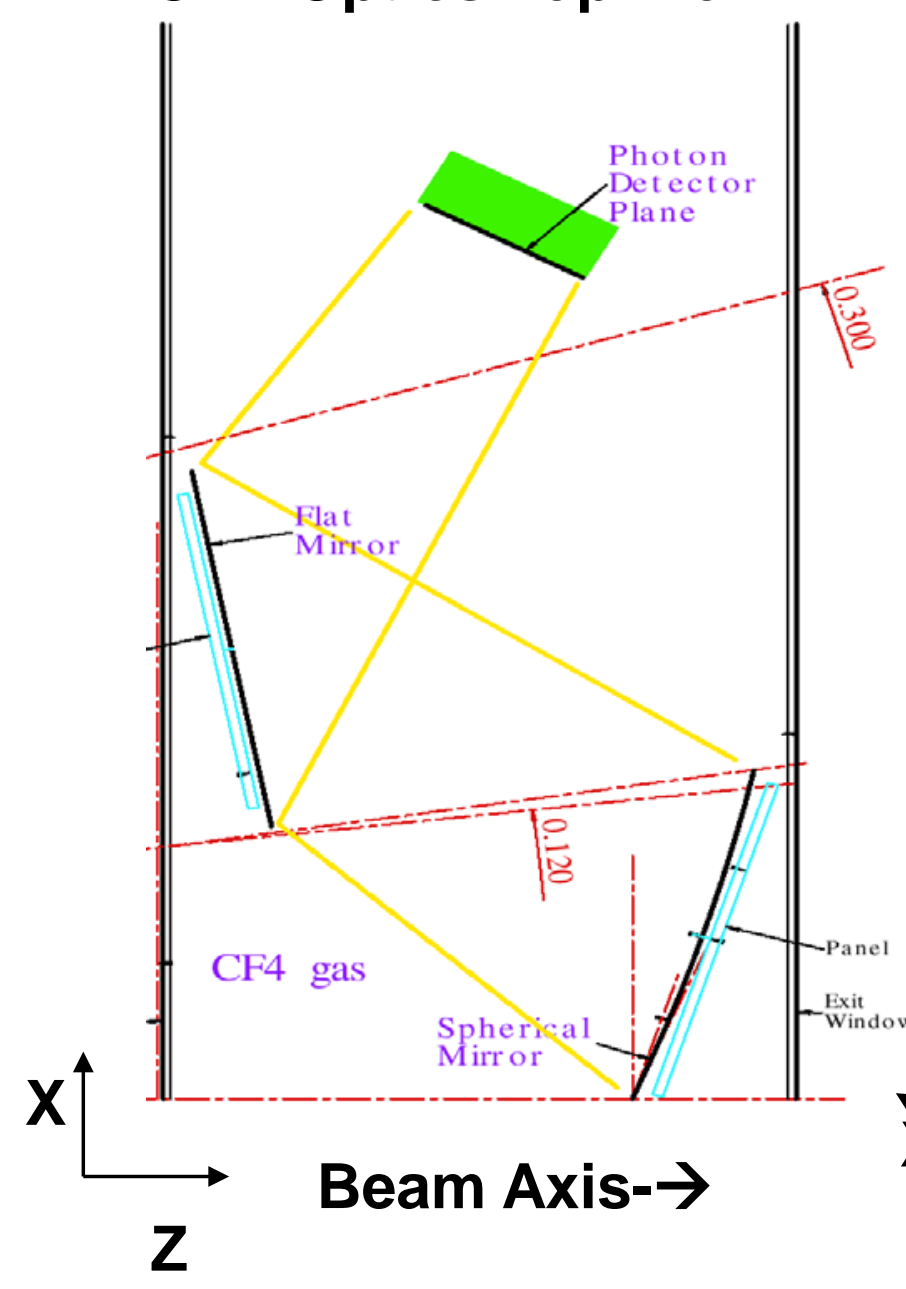

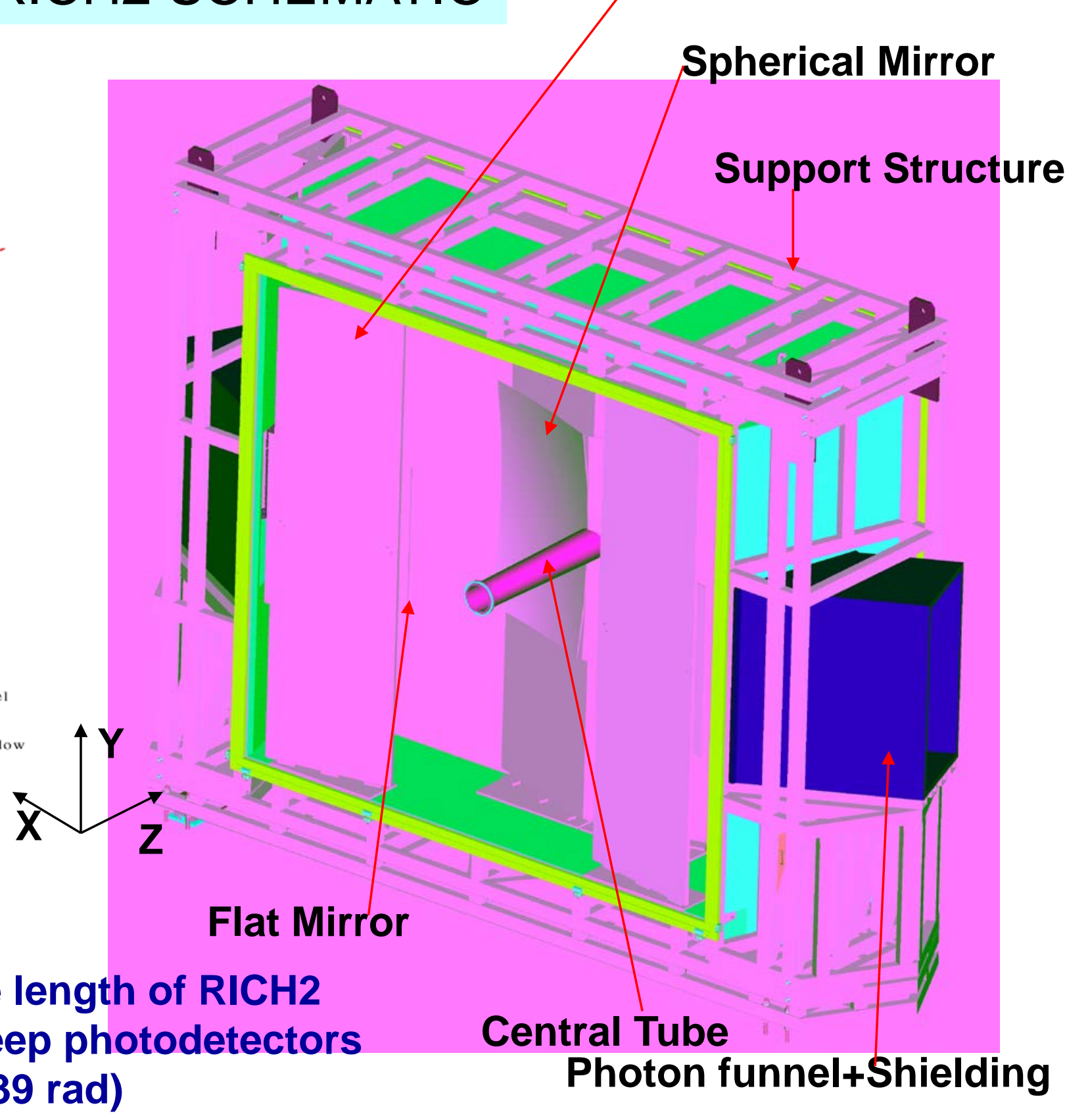

- Plane Mirrors to reduce the length of RICH2

- Spherical mirror tilted to keep photodetectors outside acceptance. $(\mathrm{til} t=0.39 \mathrm{rad})$

Mirror Support Panel

Spherical Mirror

Support Structure 


\section{RICH2 COMPONENTS}

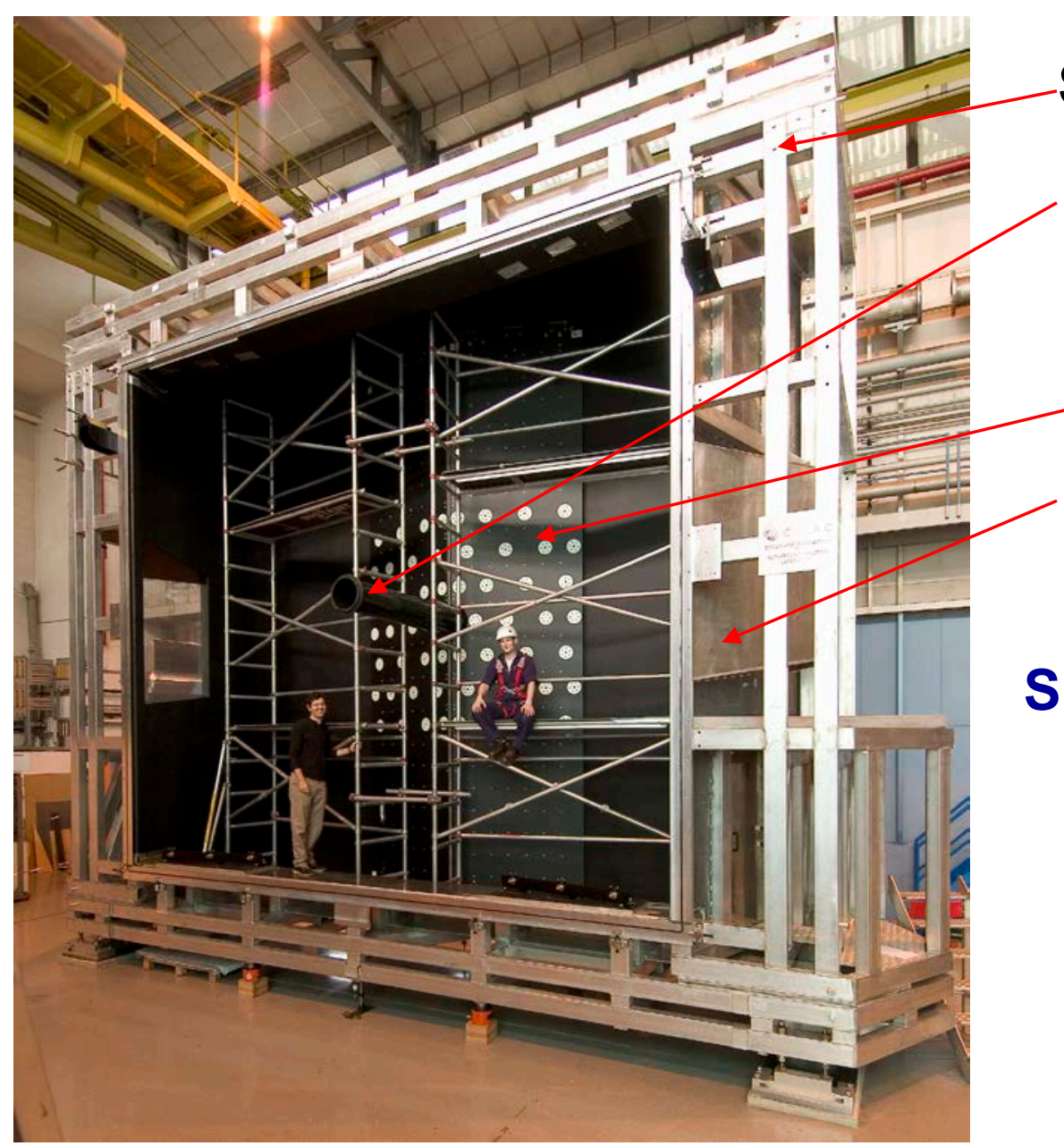

Flat mirror: 20 Rectangular segments

- size of a segment $=410 \times 380 \mathrm{~mm}^{2}$

All mirrors made of Simax (Borosilicate) glass $\mathrm{Al}+\mathrm{SiO}_{2}+\mathrm{HfO}_{2}$ coating for the required reflectivity Production of the mirrors is underway Structure (Al Alloy) Central Tube Around Beam Pipe (Carbon fibre epoxy) Mirror Support Frame Photon Funnel

Spherical Mirror: RoC $=8600 \mathrm{~mm}$ - 42 Hexagons +14 Half Hexagons - size of a hexagonal segment $=510 \mathrm{~mm}$

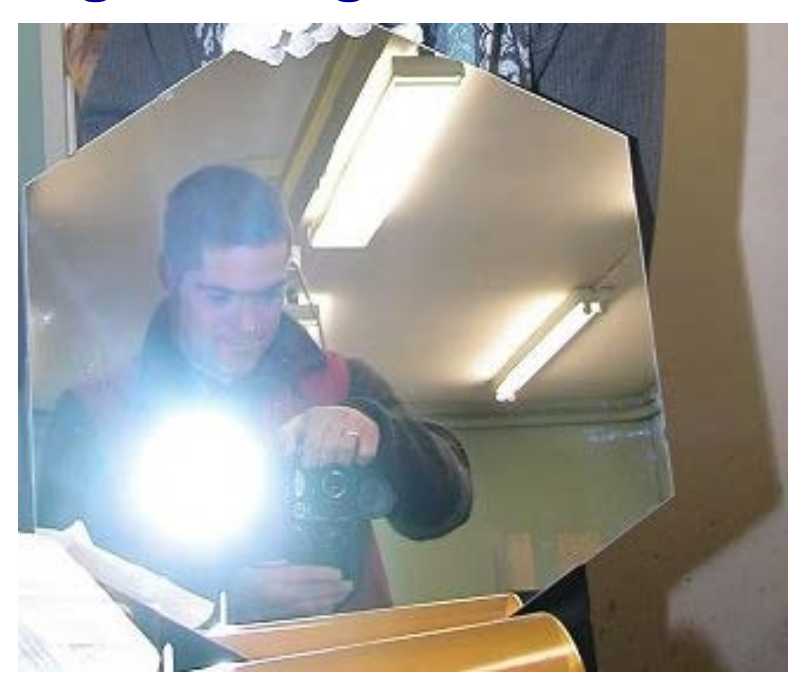




\section{RICH2 STRUCTURE ASSEMBLY}

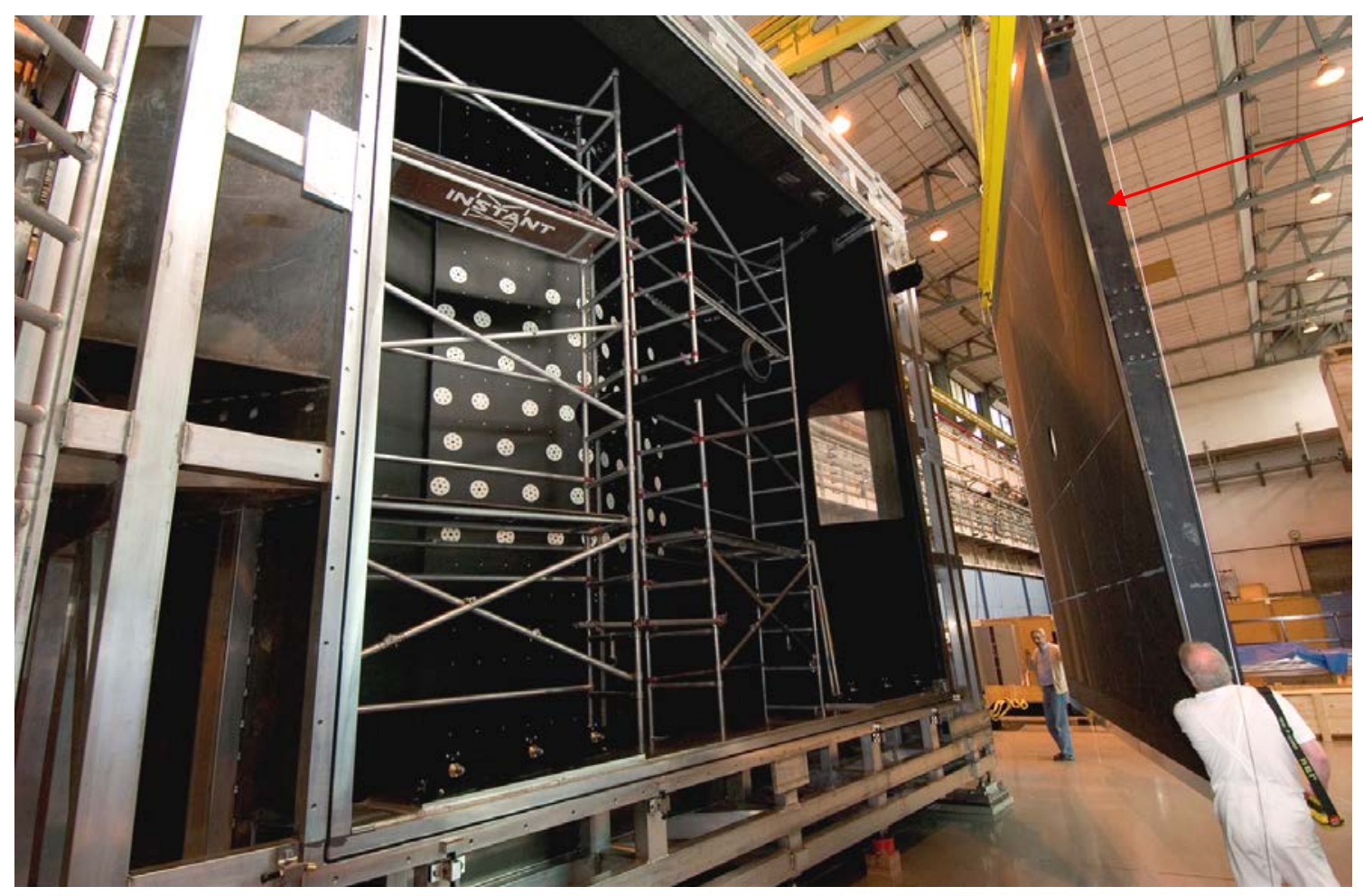

Entrance Window

(PMI foam between two carbon fibre epoxy Skins)

- Final verifications of the structure in progress at CERN

- Mirrors and Shielding to be Mounted

- Scheduled to be transported to LHCb cavern in summer, 2005

- At installation time, alignment of mirrors using a laser based system, to well below one mrad

- Monitor changes in Mirror alignment of a set of mirrors using a dedicated laser system inside the gas vessel

- Final alignment using data 


\section{Photon Detectors and Readout System}

- Active area fraction (>73\%), Granularity of $2.5 \times 2.5 \mathrm{~mm}^{2}$, Sensitive in $200 \rightarrow 600 \mathrm{~nm}, 40 \mathrm{MHz}$ readout and tolerant well beyond $3 \mathrm{~K} \mathrm{Rad} / y e a r$
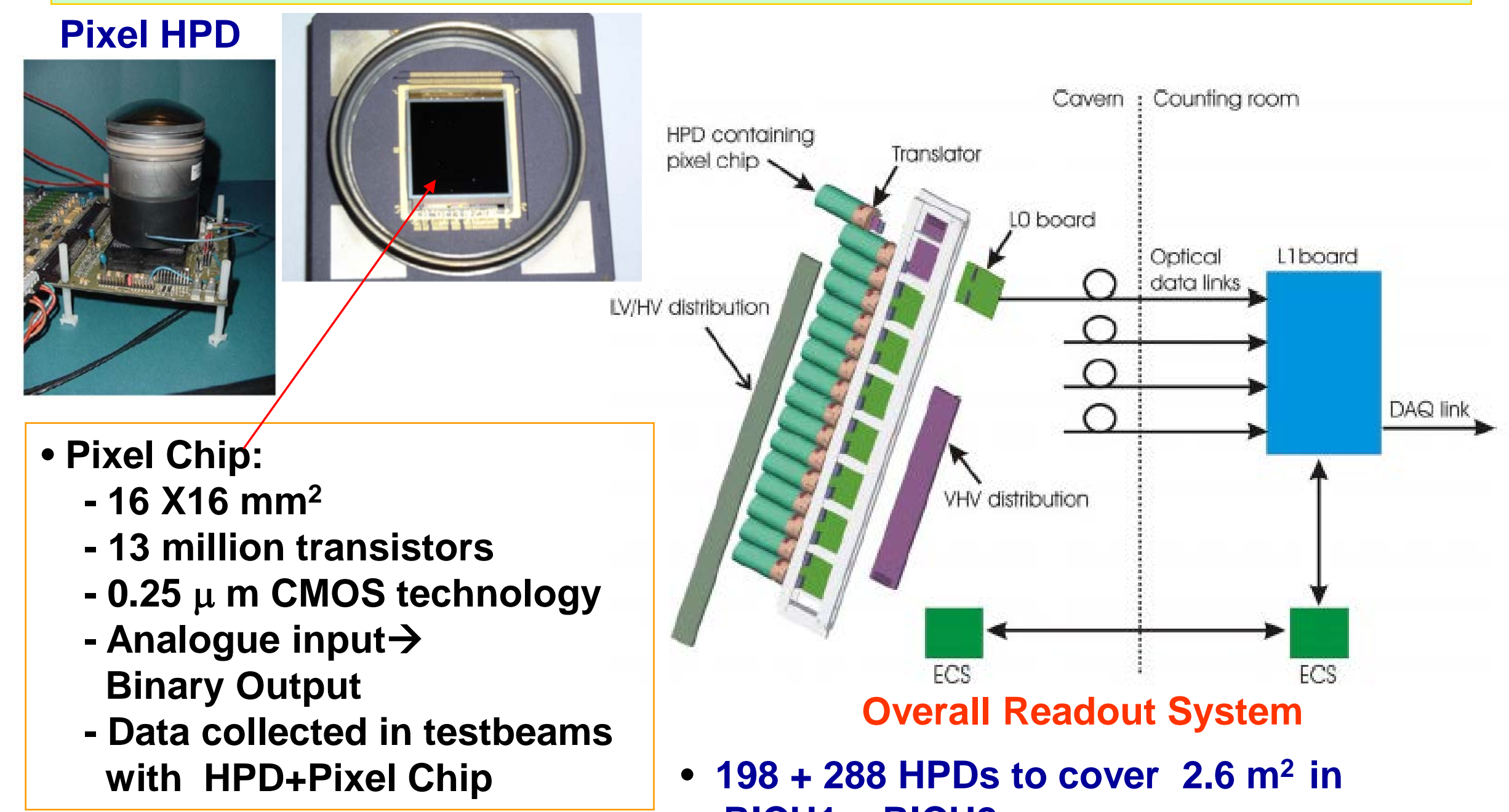

- $198+288$ HPDs to cover $2.6 \mathrm{~m}^{2}$ in $\mathrm{RICH} 1+\mathrm{RICH} 2$

- Ref. Talk by N. Kanaya on tests using HPDs 


\section{RICH SOFTWARE AND PERFORMANCE}

$>$ Detector Simulation and performance evaluation has been an integral part of RICH detector development

$>$ Using an OO Framework (GAUDI) in C++, a complete software chain implemented for all $\mathrm{LHCb}$ detectors, including the RICH

\section{Geometry in XML DB}

PYTHIA EVTGEN
GEANT4 Simulation
Digitization

Reconstruction

Physics Analysis

$>$ This facilitates detailed simulations of the Detectors

$>$ RICH Reconstruction: Reconstruct Cherenkov Angle from Hits $\rightarrow$ Global Log likelihood method.

$>$ RICH in Trigger: useful for channels like $B{ }_{s} \rightarrow D_{s}{ }^{+} D_{s}^{-} \rightarrow K^{+} K^{-} \pi^{+} K^{+} K^{-} \pi^{-}$ Offline Pattern Recognition 1s levent on 1GHz PC Online: A possible algorithm:

Fast 'likelihood method' in the Hit space

No Angle reconstruction; tests show $\sim 10$ ms/event Ref. Talk by N. Neufeld 


\section{RICH PERFORMANCE}

- Used in RICH Simulation in 2004 (DC04) Not the very final engineering design

- Yield: Mean Number of hits per saturated track (Beta $\sim 1$ ).

\begin{tabular}{|l|l|l|}
\hline Aerogel & C4F10 & CF4 \\
\hline 6.8 & 31.0 & 23.0 \\
\hline
\end{tabular}

Cherenkov Angle Resolutions

\begin{tabular}{|l|l|l|l|}
\hline $\begin{array}{l}\text { Components } \\
\text { and Overall (mrad) }\end{array}$ & Aerogel & $\mathrm{C}_{4} \mathrm{~F}_{10}$ & $\mathrm{CF}_{4}$ \\
\hline Chromatic & 2.07 & 0.80 & 0.47 \\
\hline Emission Point & 0.34 & 0.80 & 0.33 \\
\hline Pixel Size & 0.57 & 0.57 & 0.16 \\
\hline Overall RICH & 2.19 & 1.29 & 0.60 \\
\hline $\begin{array}{l}\text { Overall } \\
\text { RICH+Tracks }\end{array}$ & 2.60 & 1.60 & 0.61 \\
\hline
\end{tabular}

- All these are compatible with testbeam results

Example: For RICH2 prototype, testbeam results compared to simulations in:

NIMA 456(2001) 233-247 


\section{RICH EVENT DISPLAY}

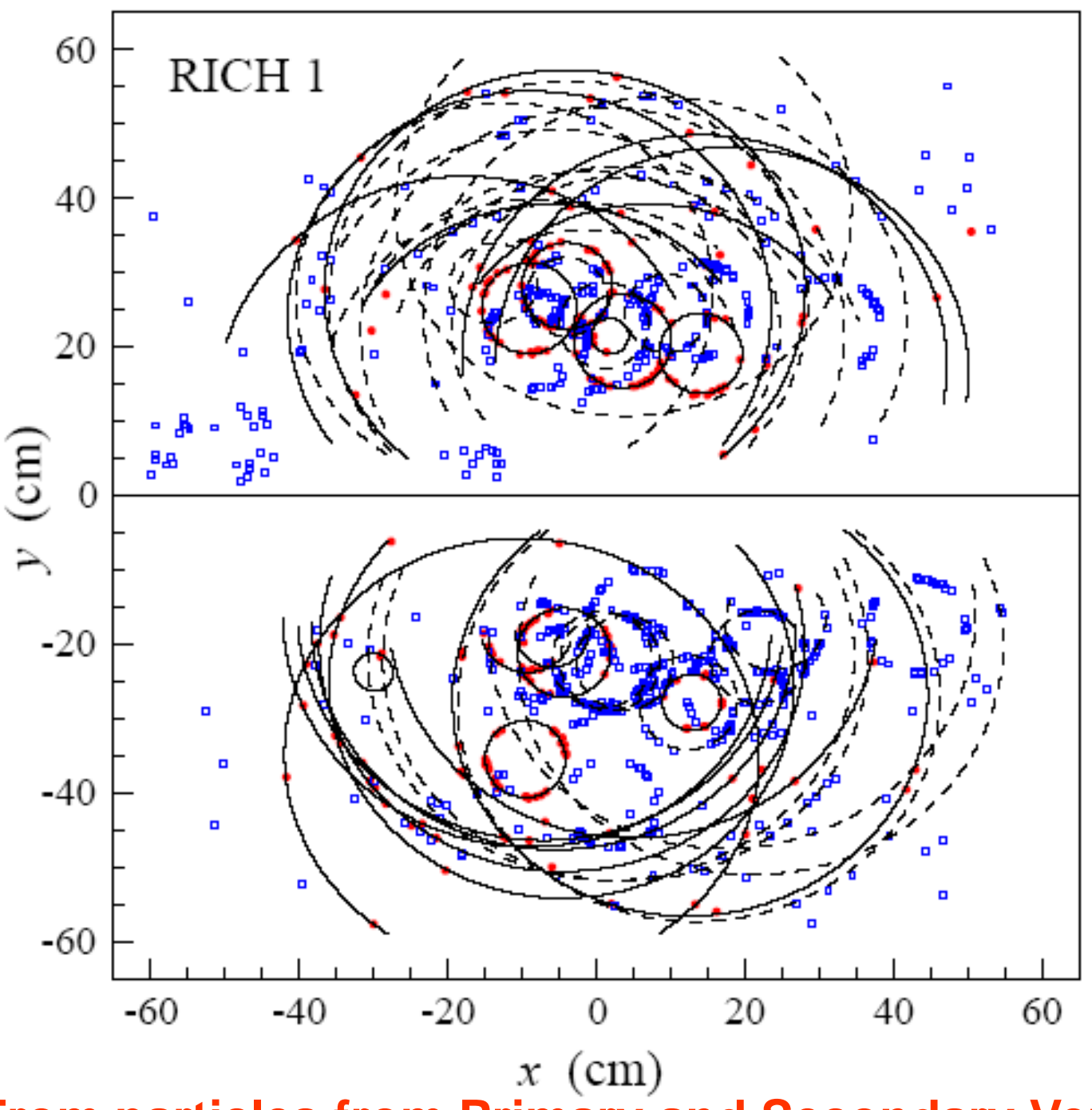

Red: From particles from Primary and Secondary Vertex Blue: From secondaries and background processes (sometimes with no reconstructed track) 


\section{RICH PATTERN RECOGNITION}
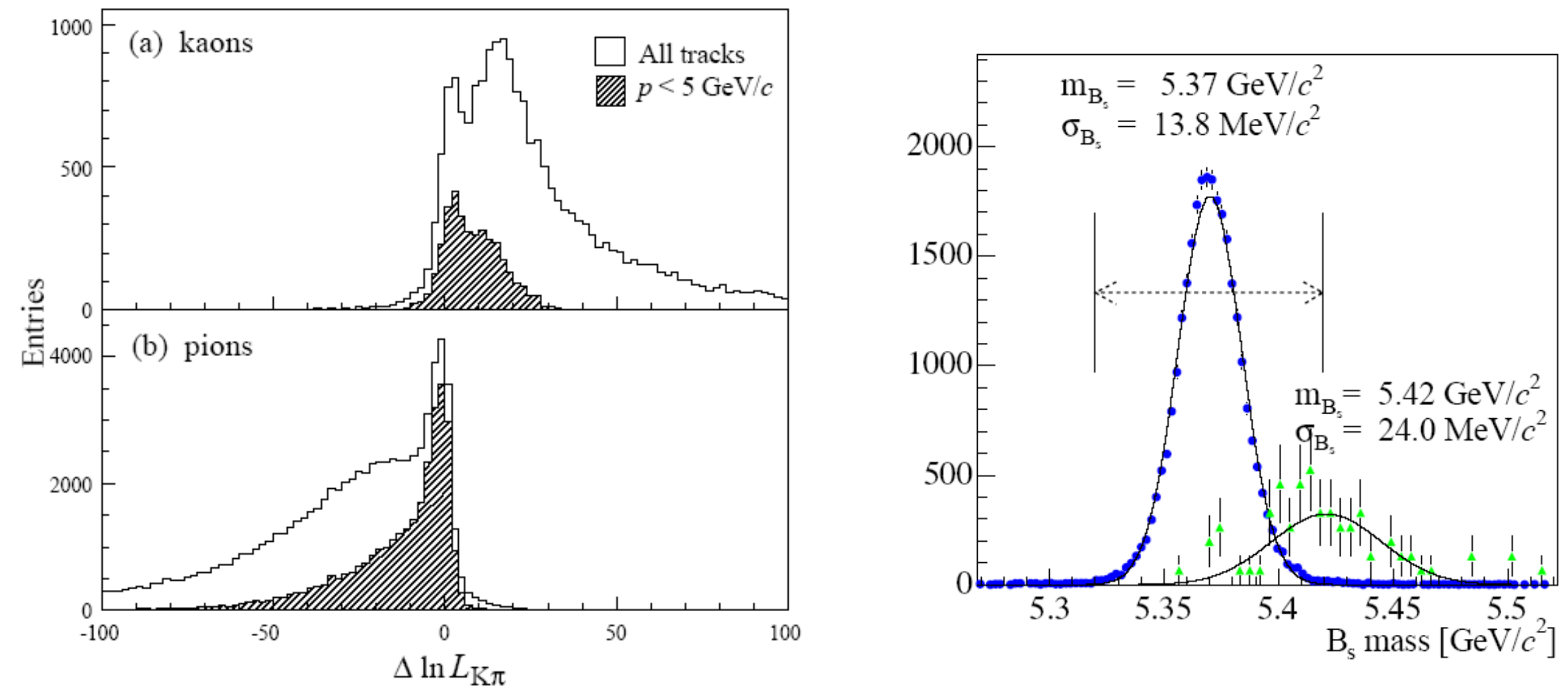

Difference in the log-likelihood between $\mathrm{K}$ and $\pi$ hypothesis in $\mathrm{B}_{\mathrm{s}}^{0} \rightarrow \mathrm{D}^{+}{ }_{\mathrm{s}} \mathrm{K}^{-}$events.

$\mathrm{B}_{\mathrm{s}}^{0} \rightarrow \mathrm{D}_{\mathrm{s}}{ }^{+} \mathrm{K}^{-} \quad \mathrm{B}_{\mathrm{s}}^{0} \rightarrow \mathrm{D}_{\mathrm{s}}{ }^{-} \pi^{+}$

(signal)

(background)

In general, $\Delta \ln \mathrm{L}_{\mathrm{k} \pi}$ is positive for kaons and negative for pions.

After using cut on difference in loglikelihood, background at 10\% level 


\section{RICH PERFORMANCE}

- After Particle Identification, Efficiency (in \%) of pion and kaon identification and Probability (in \%) of misidentifying pion and kaon for different momenta

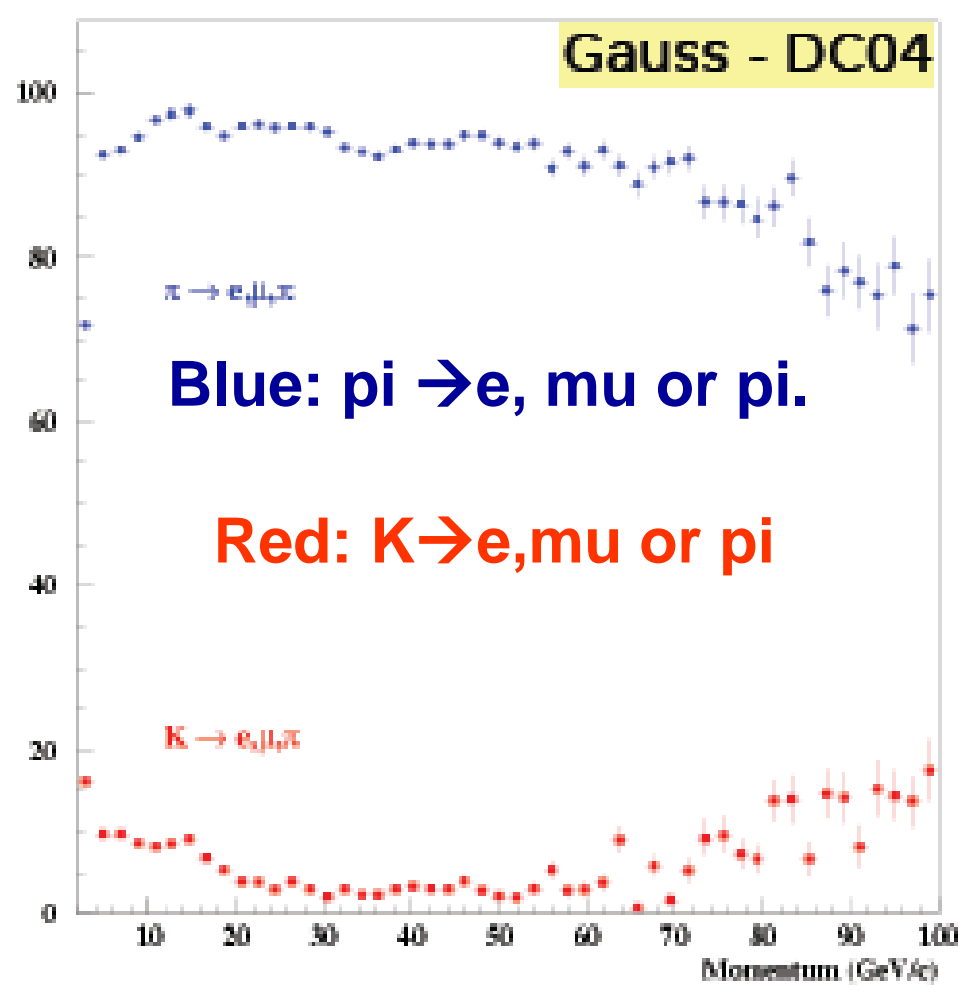

Particle Momentum (Gev/c) $\rightarrow$

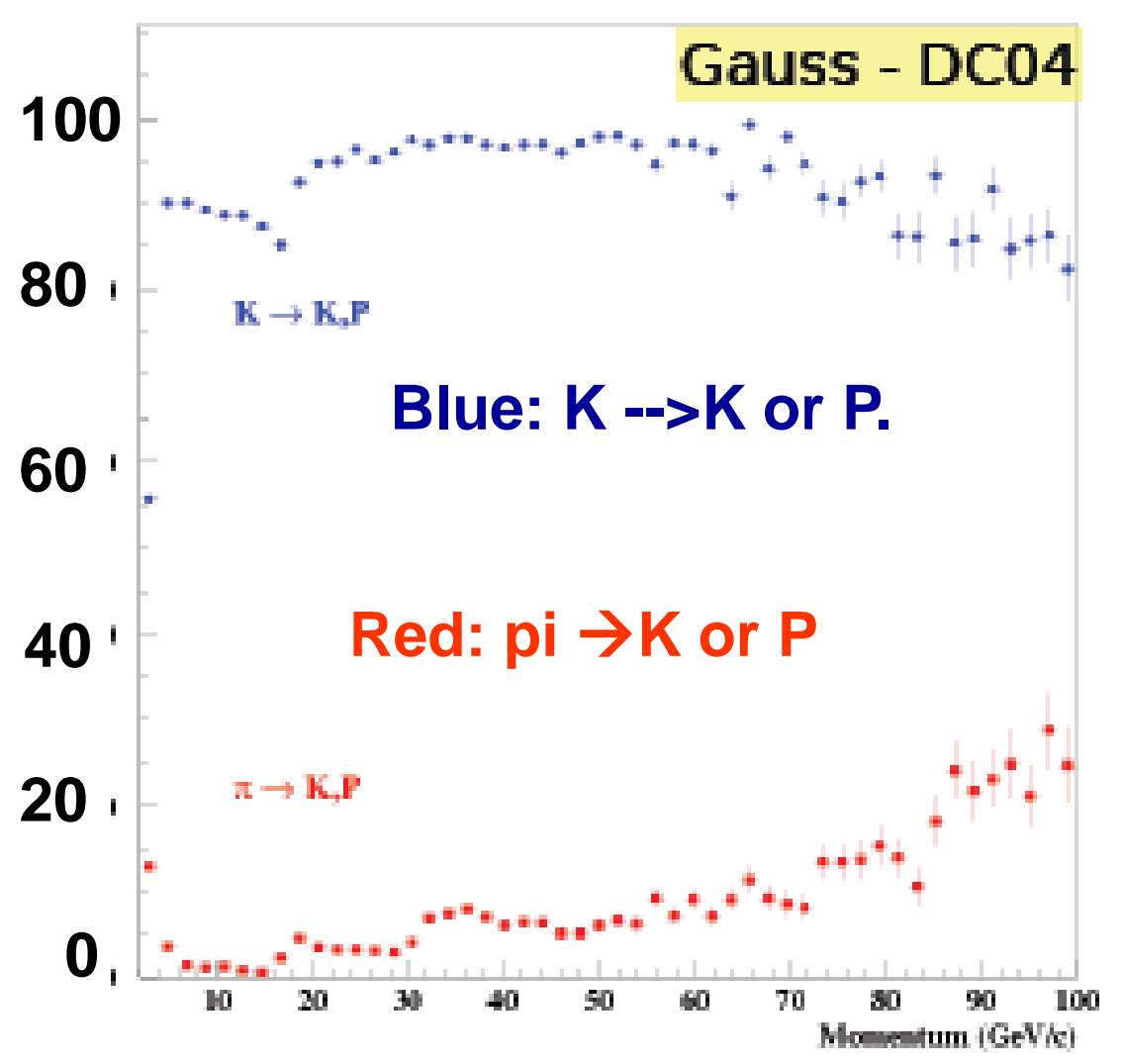

Particle Momentum (Gev/c) $\rightarrow$

- Best measured tracks in Minimim Bias events used for this 


\section{SUMMARY AND PLANS}

- RICH is an essential component of LHCb

- HPDs are now being produced for the RICH detectors

- Engineering Designs of both RICH detectors are accomplished

- RICH2 construction almost complete and RICH1 construction underway

- Installation expected to be completed by October 2006

- Detailed simulation using GEANT4 done and expected performance verified 